\title{
Gem Trade LAB NOTES
}

\section{Fused AMBER}

The Fall 1983 Gem Trade Lab Notes column reported on a necklace of beads composed of sizable chunks of amber embedded in plastic. Figure 1 shows a similar necklace that was recently submitted to the New York laboratory. However, the beads in this necklace proved to be made from chunks of amber that were somehow fused together without the use of any identifiable cement or other binder. Many of the beads exhibited black, irregular inclusions at the contact surfaces where the fragments were fused together (figure 2).

These beads were apparently manufactured by a process different from that used to produce the pressed amber that we usually see, a process by which pieces of amber are reduced to tiny fragments and then pressed through a sieve-like apparatus. This results in a fairly clear product, but one that has a "cream-of-wheat" texture when viewed with magnification. In addition, tiny bumps corresponding to the individual fragments are visible on the surface of this material in reflected light. None of the beads in the necklace shown in figure $\mathbf{1}$ had either of these characteristics.

$R C$

\section{DIAMOND}

\section{Fanciful Cuts Created by Laser Sawing}

Until just a few years ago, diamonds were parted by one of two methods: cleaving or sawing. Both of these methods have specific limitations caused by the diamond's crystalline

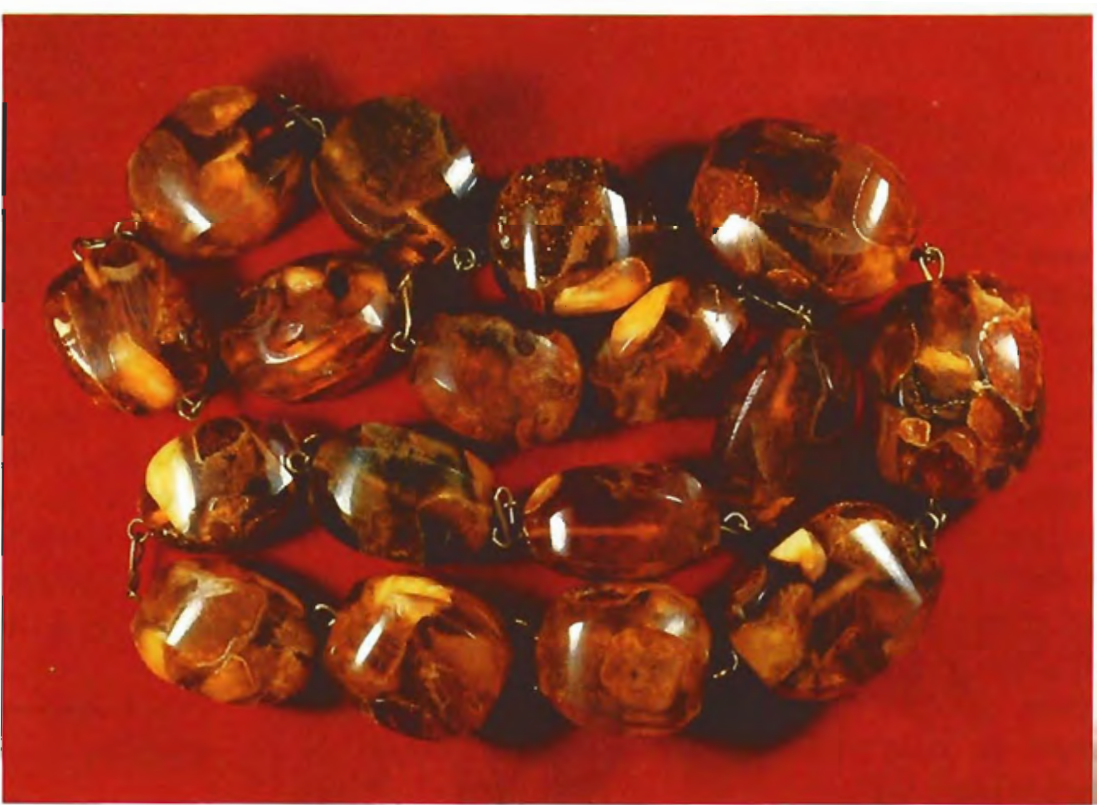

Figure 1. The beads in this necklace $(31 \times 26 \times 24 \mathrm{~mm})$ appear to be composed of chunks of amber that have been fused together.

structure, which dictates in what directions the stones may be cleaved or sawed. The somewhat recent introduction of lasers into diamond cutting has provided an effective alternative. Without applying any pressure on the diamond being fashioned, the laser can part the stone regardless of its crystalline structure, sometimes much faster and more economically than by traditional methods. Even problematic diamonds, such as those with knots or disoriented internal grains, can be laser sawn in virtually any direction. In addition, the lasser technician can cut diamond rough into predetermined designs without regard to orientation.
Figure 2. Black inclusions of an unknown nature occur at the contact surfaces of the fused fragments in the beads shown in figure 1. Magnified $10 \times$.

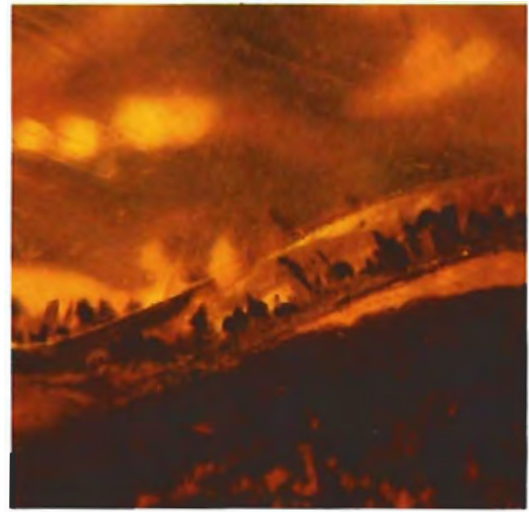


Over the past few years, diamonds have appeared in many ornate shapes, such as butterflies, fish, horse heads, trees, and flowers (the Spring 1986 Gem Trade Lab Notes shows a complete alphabet of faceted diamonds cut by laser). Generally, these fancifully cut diamonds are faceted on both crown and pavilion surfaces.

The Los Angeles laboratory recently had the opportunity to examine several interesting fancy-cut diamonds somewhat different from those seen previously, including a sail boat $(12 \mathrm{~mm}$ long $\times 2 \mathrm{~mm}$ thick) and a tennis racket matched up with a baguette diamond for the handle (figure 3). Both were cut from thin macles, thereby producing relatively large stones from difficult to cut pieces of rough. For each item, the large parallel faces of the macle were first polished flat and the outline of the shape cut with a laser. The grooves were then cut in to the back of the piecd, and facets were placed around the edges on both the top and the bottom. These diamonds are somewhat reminiscent of the "profile cut" diamonds introduced in 1961.

$R K$

\section{Natural-Color Light Green Diamonds}

Although the presence of small green or brown irradiation stains on the surface of light green diamonds (usually in or near naturals) does not automatically prove that the color is natural, it is a strong indication. We know of no such stains being produced artificially. It is important to note, however, that while these irradiation stains are most probably of natural origin, they do not prove that the "body color" of the diamond is entirely of natural origin. Near-colorless or faint green diamonds with

Editor's Note: The inilials at the end of each item identify the contributing editor who provided that item.

(C) 1986 Gemological Institute of America

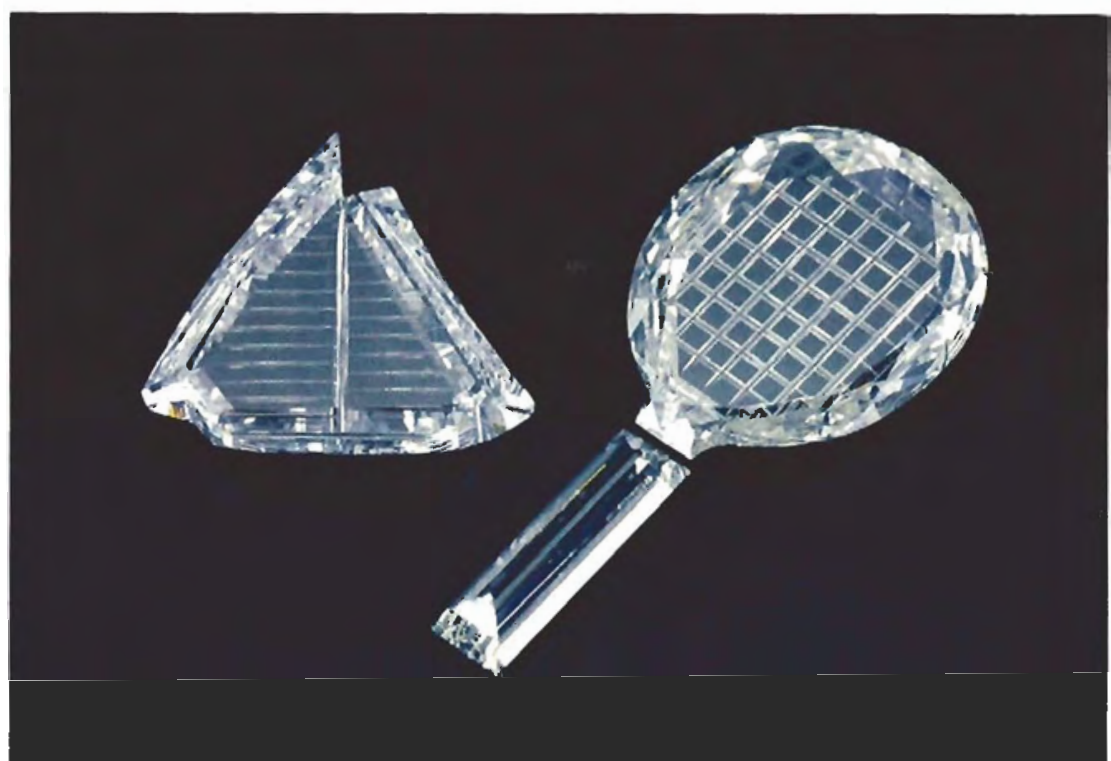

Figure 3. With the technology provided by laser sawing, fanciful cuts such as this 12-mm sailboat and tennis racket can be obtained from otherwise difficult to cut pieces of rough.

such stains could be artificially irradiated to darken the color or produce a light to dark green color.

Sometimes the heat of polishing will alter the green "skin" color to brown. This evidently occurred on the stone shown in figure 4, where a tiny brown natural irradiation stain may be seen at the culet. The stone was graded "very light green," with a statement to the effect that the presence of an irradiation spot indicates natural color.

Nearly all diamonds cut from the green "speculative" rough sold by the Diamond Trading Company are considered speculative because they usually do not remain green after being cut; rather, they end up being in the near-colorless " $\mathrm{G}-\mathrm{H}^{\prime}$ range. The thin green "skin" effectively masks the true body color. Very rarely, one of these stones turns out to be faint to light green even if all surface evidence of the green skin is removed. Since the color is due to irradiation and, in most cases, no tests exist to distinguish between natural and man-induced irradiation, stones of this color but without a green- or brown-stained natural are saddled with a "color origin unde- termined" report. On occasion, the laboratory has advised cutters to leave a little of the colored surface on the finished stone if it is one of the rare pieces of green rough that retains some of the color after cutting.

One example of green surface irradiation staining that left no doubt as to the natural origin of the surface color is shown in figure 5. The 2.35ct rough crystal was very kindly loaned to us for study by a New York diamond dealer. It is difficult to imagine what conditions within the

Figure 4. Irradiation stains on green diamond rough are sometimes altered to brown, as seen here, by the heat of polishing. Magnified $20 x$

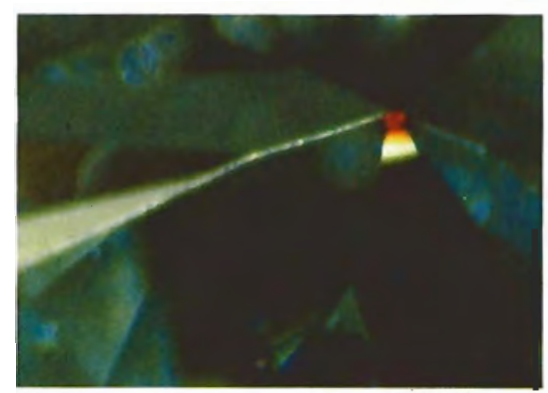


earth could have caused this dendritic surface pattern. The rough would probably cut a near-colorless stone, although it is hoped that it will not be cut, since in its present state it is a true phenomenon of nature. $R C$

\section{Unusual Inclusion in Diamond}

The same dealer provided us with another "mind blower" in the form of a sawed diamond crystal fragment. Looking through the stone toward the sawed area, one can see what appears to be a large green crystal inclusion that was obviously the reason for dividing the diamond at that point. However, upon turning the piece over, one sees that the "included crystal" is really a negative crystal with a green lining (figure 6). To add even more to the uniqueness of this specimen, inside the greenlined negative crystal is a tiny green diamond crystal that is completely loose within the cavity.

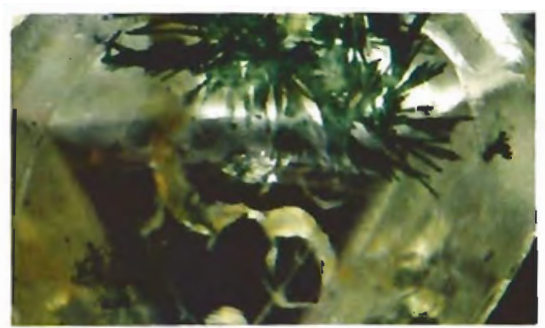

Figure 5. This unusual green dendritic irradiation stain was observed on the surface of a 2.35-ct diamond crystal. Magnified $10 \times$.

Figure 6. A loose green diamond crystal is trapped inside a green irradiation-stained cavity in this sawed diamond section. Magnified $15 \times$.

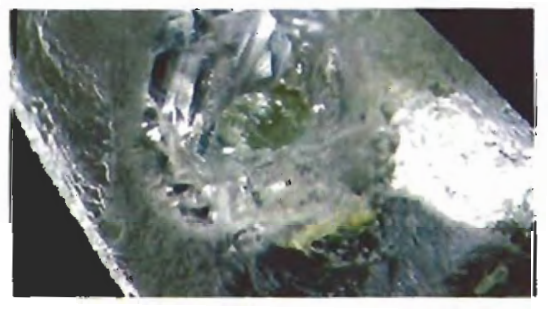

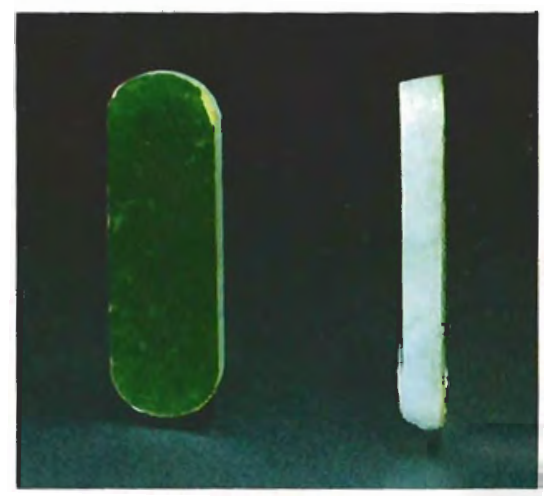

Figure 7. This $20 \times 6 \times 2.5 \mathrm{~mm}$ assembled tablet consists of a thin $(0.1 \mathrm{~mm})$ layer of green iadeite on top of a thicker (2.2-2.3 mm) layer of white jadeite, as evident in this double-exposure photo.

\section{Jadeite JADE Doublet}

Figure 7 shows an oval tablet $120 \times 6$ $\times 2.5 \mathrm{~mm}$ ) that the Los Angeles lab received for identification. Simple observation revealed that the tablet was assembled. The piece consisted of two parts, one extremely thin (only $0.1 \mathrm{~mm}$ ) green layer at the top and another much thicker $\{2.2-2.3$ $\mathrm{mm}$ ) white layer at the bottom. Using high magnification, we noted that the layers had been joined by a slightly yellowish cement that contained numerous small gas bubbles (figure 8). The thin top layer was a mottled dark green with numerous small near-colorless veins (figure 9). Visually, this part of the assemblage somewhat resembled green chloromelanite. The white bottom portion showed a definite crystalline structure. We were able to obtain indistinct R.I. readings (ranging from 1.64 to 1.74 ) on the green portion, which seemed unusual. When examined with a spectroscope in both reflected and transmitted light, the green layer revealed distinct lines in the red portion of the spectrum, suggesting that it was colored by chromium. Although the tests indicated that the top was probably natural-color jadeite jade, we could not positively identify both layers with ordinary

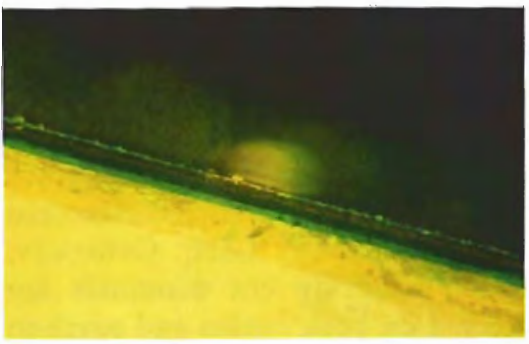

Figure 8. At $63 \times$ magnification, the yellowish cement that joins the two jadeite layers shown in figure 7 is visible.

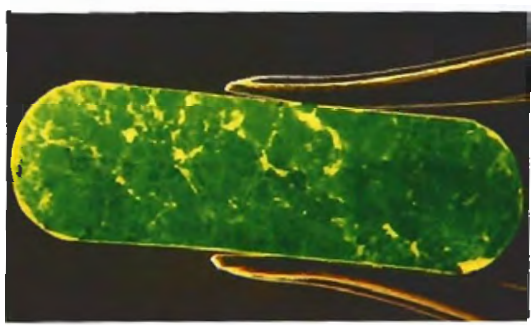

Figure 9. The top portion of the assembled jadeite piece is a mottled green with numerous small near-colorless veins. Magnified $10 \times$.

gemological testing methods. Therefore, we tested both the green and the white portions on the infrared spectrometer. The infrared spectra of both portions matched the spectrum of jadeite jade. We also scraped a tiny amount of powder from each layer for X-ray diffraction analysis. The diffraction patterns obtained for both layers matched that of jadeite jade, strongly indicating that both parts of the assembled stone were jadeite jade. However, at the present time we have no explanation for the apparent variation in the refractive indices of the green portion. Further analysis would be desirable. $K H$

\section{Dyed LAPIS LAZULI, Difficult to Detect}

The Los Angeles laboratory was recently asked to determine whether or not the lapis lazuli necklace illustrated in figure 10 had been dyed. Ev- 


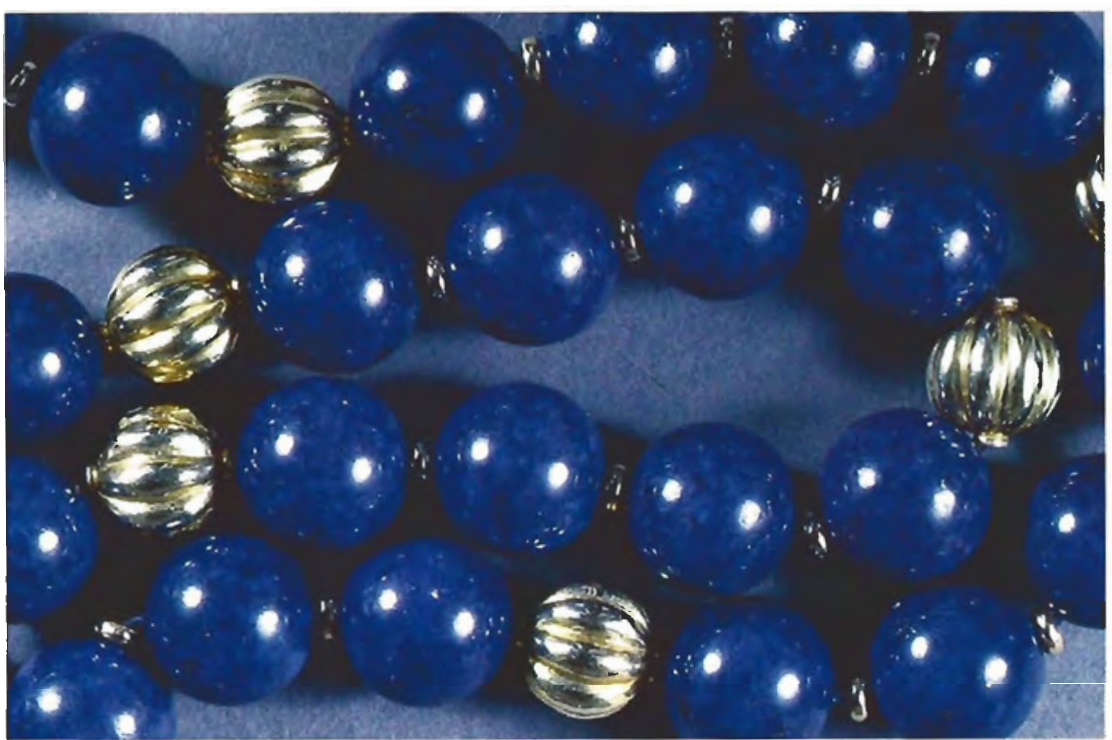

Figure 10. The dye in these 15-mm lapis lazuli beads could not be detected on an acetone-soaked swab, but did show when the beads were carefully tested with hydrochloric acid.

idence of dye is most frequently encountered as a concentration of blue or purple in the fractured and porous areas. This necklace had an unusually large number of distinctly purple areas, which were easily seen with the unaided eye. However, when the beads were rubbed first lightly and then vigorously) in an inconspicuous area with an acetonesoaked cotton swab, no dye appeared on the swab. Since lapis lazuli is sometimes coated with wax to hide dye, conceal a poor polish, or prevent dye from staining clothing or stone papers /see the Summer 1981 and Summer 1986 Gem Trade Lab Notes), we looked for a wax by careful microscopic examination and testing with a hot point. None was revealed.

Several years ago, the laboratory examined a dyed blue marble bead which when tested with acetone did not show any evidence of dye. However, when that marble bead was tested with a solution of $10 \%$ hydrochloric acid, a very dark blue stain appeared on the cotton swab (see the Fall 1982 Gem Trade Lab Notes). We decided to test the lapis necklace shown in figure 10 with this method.
Using a cotton swab soaked with a $10 \%$ solution of hydrochloric acid, we obtained a light bluish purple stain. Testing of only the purple areas yielded more of a stain than did other areas of the beads. Therefore, if no evidence of dye is obtained with acetone, one should also try the dilute $\mathrm{HCl}$ solution before concluding that there is no evidence of dye. As always when handling acids, use extreme care with $\mathrm{HCl}$.

$R K$

\section{PEARLS}

\section{Black Cultured Pearls}

When natural-color black cultured pearls from Tahiti and, more rarely, from the Philippines and Okinawa, first appeared in the trade, the GIA Gem Trade Laboratory tested them with long-wave ultraviolet radiation. By comparing the fluorescence color with that of a polished shell from Tahiti, we became familiar with the characteristic indistinct red-brown glow of the natural-color black cultured pearls. We were also impressed with the distinct appearance of these cultured pearls in the radiographs. We rarely have any difficulty recog- nizing them as cultured since there is almost always a clear-cut difference between the nacre and the nucleus. In fact, they are often divided by a black ring that is caused by the transparency of the conchiolin layer surrounding the nucleus (figure 11).

In the past year or so, we have seen an increasing number of treated black cultured pearls. These treated black cultured pearls ei ther fluoresce slightly greenish (rather than redbrown) to long-wave ultraviolet radiation, or are completely inert. Also, necklaces of these treated cultured pearls are usually uniform in fluorescence, in contrast to the differing tones of their naturally colored counterparts. The greatest difference between the two products is in their $\mathrm{X}$-radiographs. Sometimes difficulties are presented in telling whether the treated pearls are cultured or natural, since the treatment effectively masks the contrast between the nacre and the nucleus (figure 12). In some necklaces, not even one black ring is visible around a nucleus. If a silver-based or other metallic dye is used, some of the pearls may show an opaque white reversal ring part way around the nucleus, since the metal deposit does not transmit X-rays.

$R C$

Figure 11. A black ring of

conchiolin between the nacre and the nucleus on this $X$-radiograph identifies these natural-color black pearls as cultured.

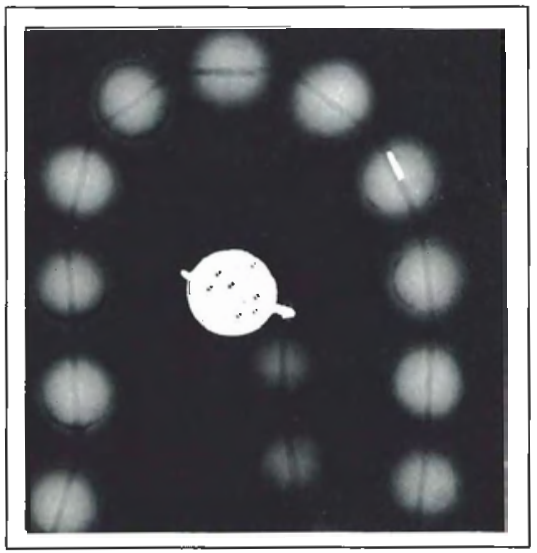




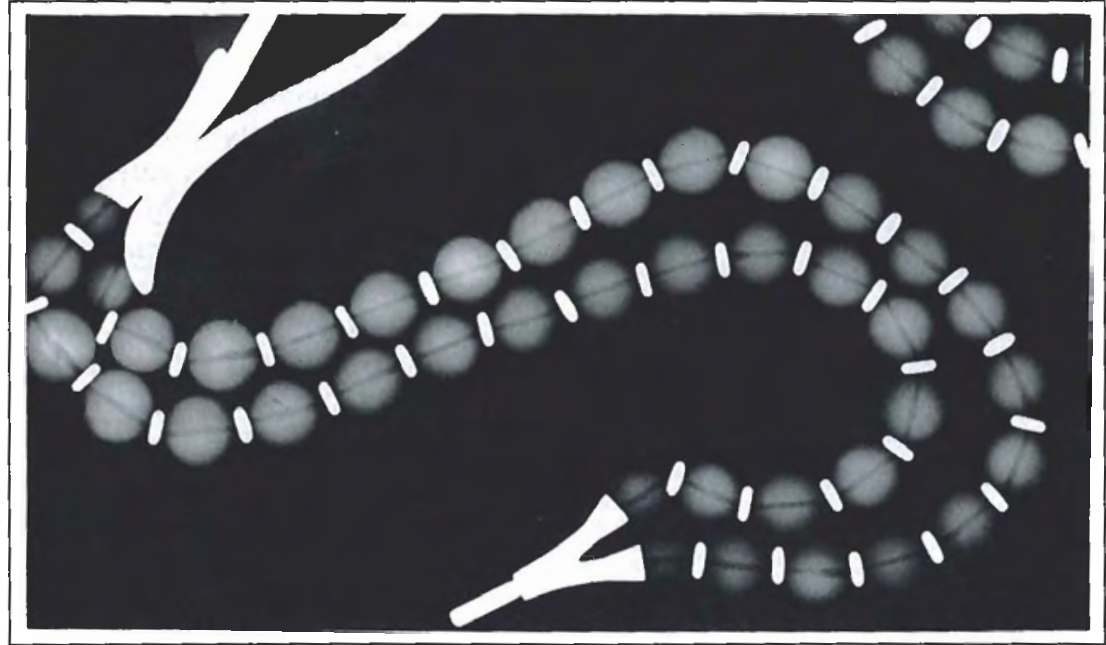

Figure 12. In these black cultured pearls, treatment has effectively masked the black ring around the nucleus on the X-radiograph.

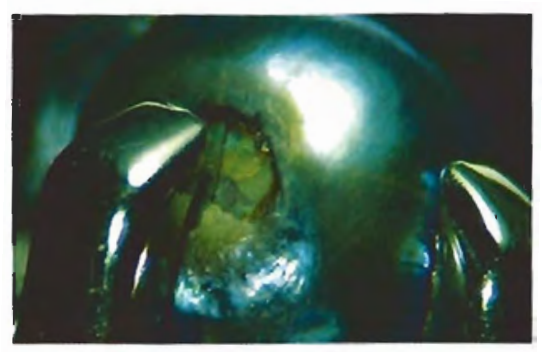

Figure 13. By examining the damaged area next to the prong on this 5-mm black pearl, the lab was able to determine that the color was natural.

\section{A Black Natural Pearl with Unusual Fluorescence}

A woman's ring set with one round undrilled pearl was sent to the Los Angeles laboratory for identification. The pearl was rather small (approximately $5 \mathrm{~mm}$ in diameter), but had a very attractive brownish black color with a fairly high luster. Even without magnification, a small damaged area was visible next to one prong (figure 13). Here the top layer was gone, exposing part of the conchiolin layer and the underlying nacreous layer, which appeared to be a deeper black and have a higher luster than the outer layer. We also noted that the outer layer was completely transparent. We concluded that the black pearls usually show a reddish brown glow when exposed to longwave U.V. radiation. Therefore, we wcre quite surprised to see that this pearl fluoresced a strong yellow. This was the first time that we have seen this remarkable color fluorescence in a natural-color black pearl. $\quad K H$

\section{Blue to Gray Saltwater}

\section{Cultured Pearls}

Recently, three $10-\mathrm{mm}$ round gray "pearls," two in earrings and the third in a brooch, were submitted to the New York laboratory (figure 14) along with several pieces that were found to contain natural pearls. These gray pearls had the appearance we have long associated with cultured pearls, whether natural color, dyed, or irradiated. However, we were surprised by the X-radiograph, which showed that each pearl had an opaque center within a shell of average-thickness nacre. The owner gave us permission to remove the pearl from the brooch, which enabled us to see by looking down the drill hole that the central material was white (figure 15) and had superficial properties much like those of the traditional French pearl cement, in-

Figure 14. All three of the 12-mm gray cultured pearls in this pair of earrings and matching brooch were found to have hollow centers filled with some foreign material.

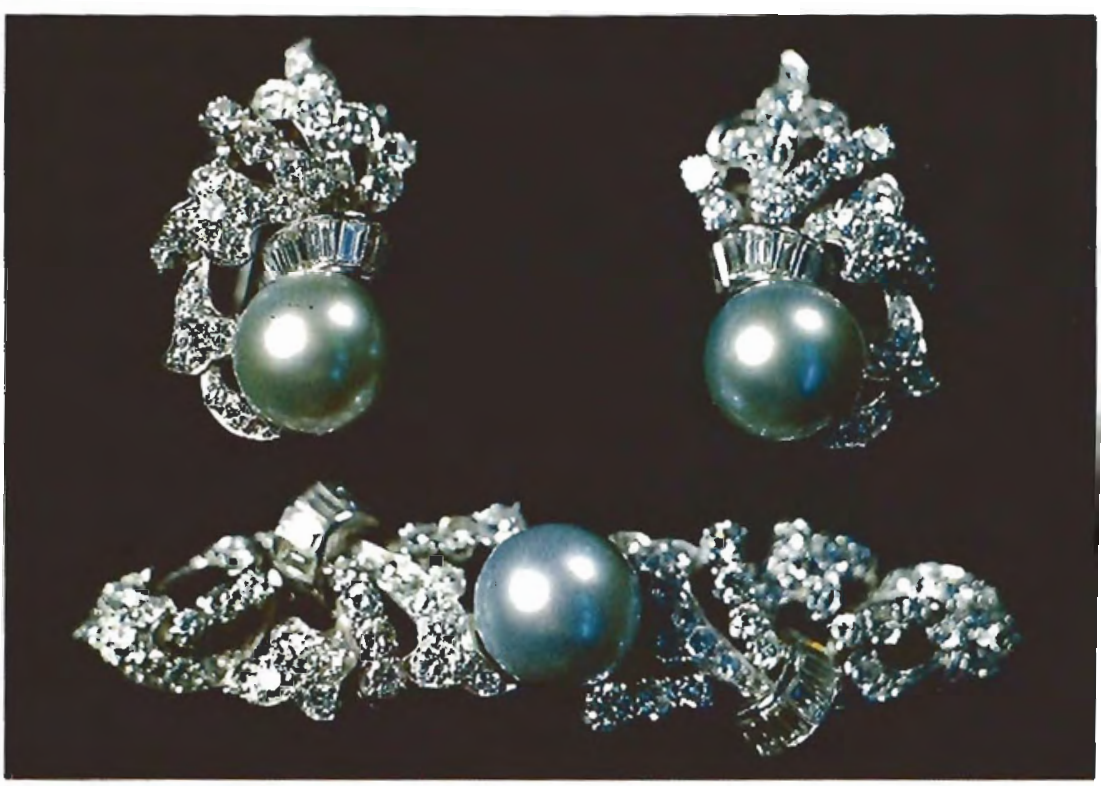


cluding the tendency to be slightly soluble to our X-ray immersion fluid.

We are mystified as to how these pearls were formed. If they are indeed cultured, what was the nucleus and how was it removed? As the photograph indicates, the drill hole through which the nucleus would have had to have been removed is an opening only approximately four times the size of a normal drill hole. Possibly, the pearls were cultured around a plastic bead or some experimental material, which was later dissolved. The coincidence of having three nearly identical hollow natural pearls is too great to be believed.

Blue to gray saltwater cultured pearls may owe their color to one or more of five different factors: (1) precolored bead nuclei that show through a transparent, fairly thin, uncolored nacre may be used; (2) growth conditions may color the nacreous layer, at times so strongly that bleaching will not remove the color; (3) growth conditions may cause an abnormal deposit of dark conchiolin around the bead nucleus before growth of the nacre begins; (4) cultured pearls (usually drilled) may be dyed, with the dye commonly lodging in the zone around the nucleus and under the nacre; and (5) they may be irradiated, an operation that darkens the freshwater shell bead nucleus under the colorless nacre.

Figure 15. A white cement-like material can be seen in the drill hole of one of the cultured pearls shown in figure 14. Magnified $15 \times$.

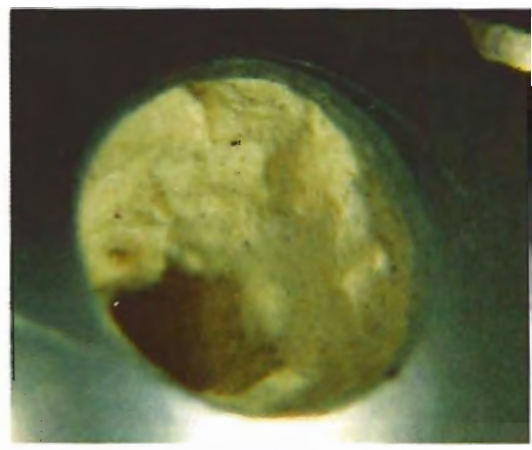

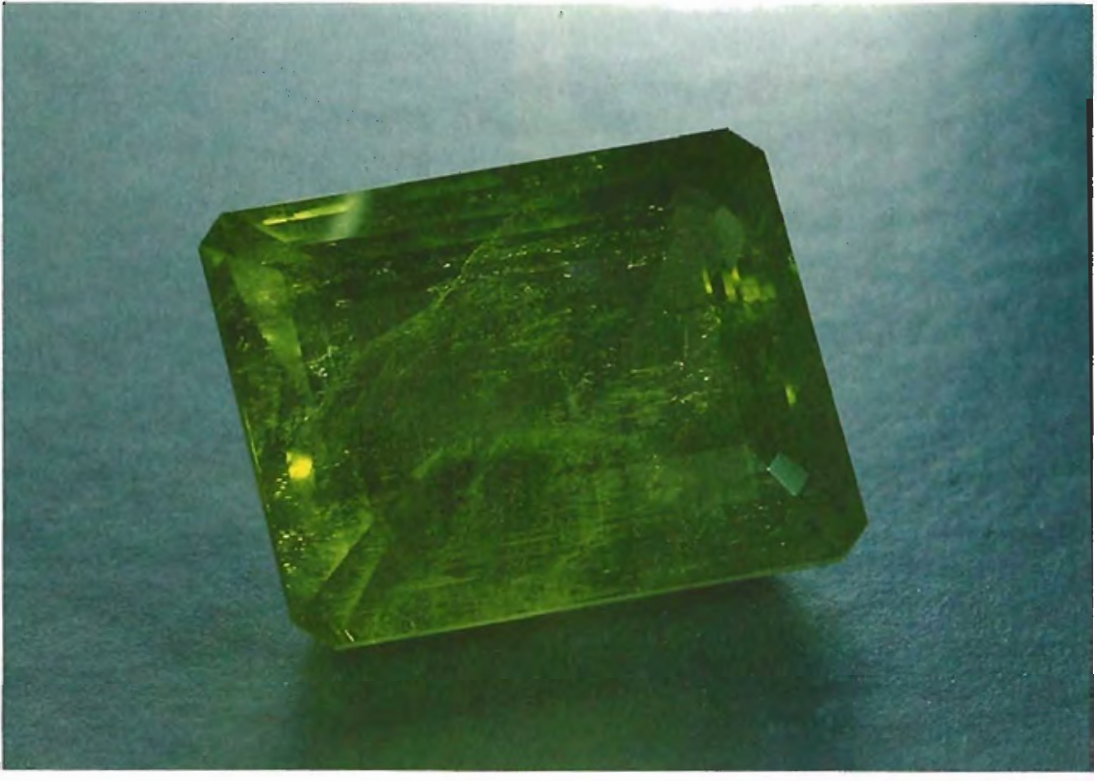

Figure 16. The yellowish green color of this 38.47-ct tourmaline is very unusual.

As seen in figure 15, the nacre is blue-gray, which indicates that the color is either natural or due to dye, but it is definitely not caused by irradiation or by a dark layer of conchiolin. As Dr. Kurt Nassau observed a number of years ago, gamma irradiation of saltwater cultured pearls darkens only the freshwater bead nuclei, not the nacre. Evidently, natural saltwater pearls or shell will not be affected by irradiation, while freshwater shell will be colored, as noted by R. T. Liddicoat, Jr. (Gems e) Gemology, Spring 1967).

$R C$

\section{TOURMALINE, Unusual Yellowish Green}

An interesting 38.47-ct yellowish green faceted tourmaline (figure 16 ) was submitted to the Los Angeles laboratory for identification. In addition to its intriguing vivid color, which until recently had not been seen in gem tourmalines, this stone gave a birefringence value of 0.028 , which is well above the maximum typically noted for other gem tourmalines $(0.020)$. However, the refractive index values of 1.621 and

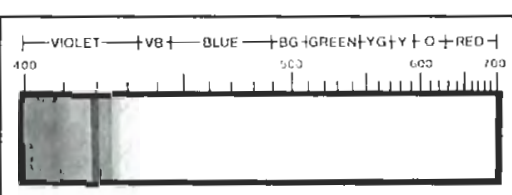

Figure 17. The absorption spectrum of the tourmaline shown in figure 16 is probably due to the unusually large amount of manganese in this stone.

1.649 and a specific gravity of 3.13 are still well within the range for the various species of tourmaline. The stone is inert to long- and short-wave ultraviolet radiation. Viewed with a GEM spectroscope unit, the stone displayed a cut-off at about $410 \mathrm{~nm}$, a strong absorption band from approximately 418 to $420 \mathrm{~nm}$, and a fainter narrow band at approximately 423.5 nm (figure 17). These bands are probably associated with manganese. The stone contained liquid-and gas-filled inclusions typical of many tourmalines (figure 18).

Chemical data from electron microprobe analysis of four separate spots on the stone revealed an unusually high level of manganese 19.2 wt.\% $\mathrm{MnO}$. Otherwise, the results 


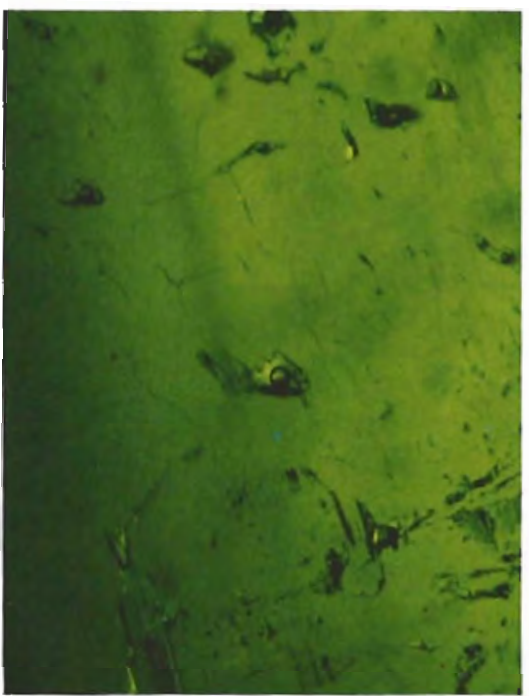

Figure 18. Two-phase, liquid and gas, inclusions similar to those found in many other tourmalines were observed in the stone shown in figure 17. Transmitted light, magnified $25 x$.

are consistent with published tourmaline analyses.

We do not know the exact origin of this particular gem tourmaline, but we have seen some material, vaguely reminiscent in color as well as in physical and optical properties, that originated from pegmatite localities along the Luangwa River Valley between Kafue and Chirundi, in Zambia. The partial resemblance to the faceted stone described here leads us to believe that it is of $\mathrm{Zam}$ bian origin.

Although some gem tourmaline from Zambia is heat treated to change or improve the color /see figure 191, we are convinced that this stone was not heat treated because of the absence of any obvious damage to the numerous liquid-gas inclusions (again, see figure 18).

In most green tourmaline, the dominant coloring agent is thought to be iron. However, Schmetzer and Bank INeues lahrbuch für Mineralogie Monatshefte, 1984, pp. 61-69) suggested that the spectral features of the yellow to yellowish brown or greenish yellow Zambian

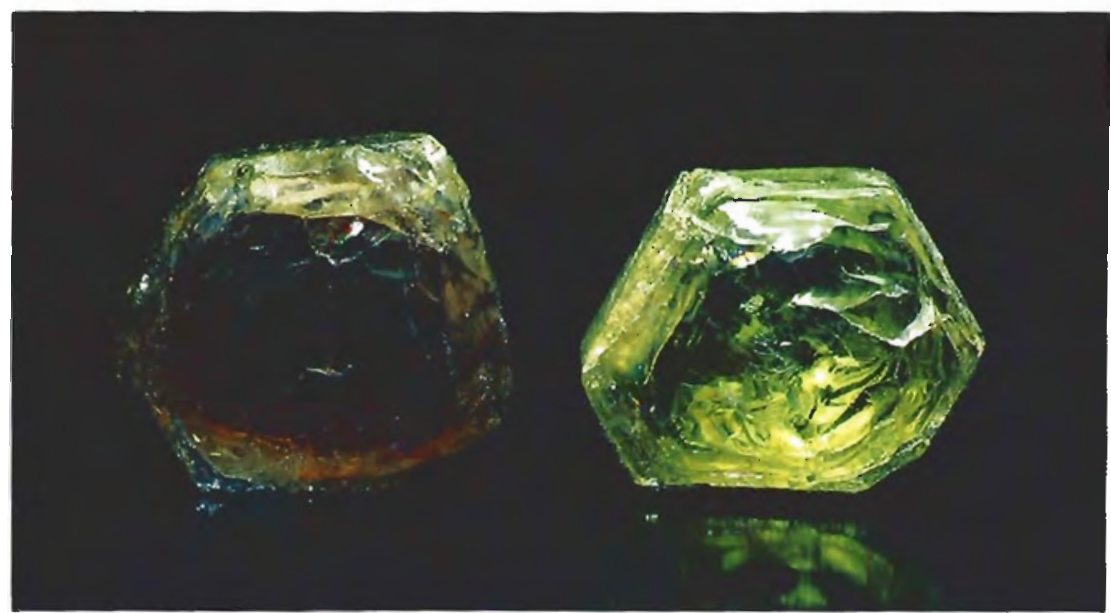

Figure 19. Reddish brown Zambian tourmalines (left) are often heated to produce a yellowish green color (right).

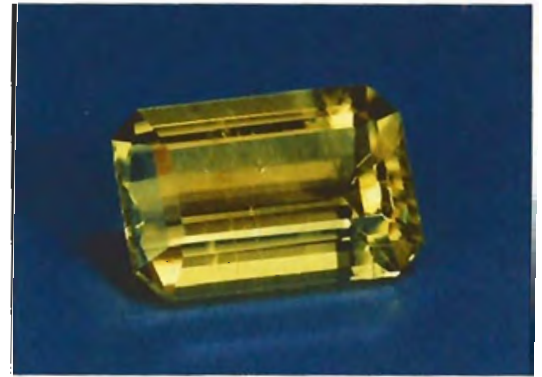

Figure 20. K. Schmetzer and $H$. Bank found an unusually high weight percentage of manganese in the greenish yellow Zambian tourmalines that they studied, which are similar in color to the stone shown here.

tourmalines that they studied (see figure 20| may have involved manganese. Tourmalines in general contain no more than a few weight percentages of $\mathrm{MnO}$, but Schmetzer and Bank reported $6.37-6.80$ wt. $\% \mathrm{MnO}$ in the Zambian tourmalines they studied.

In 1929, Kunitz /Chemie der Erde, pp. 208-251) proposed the name "tsilaisite" for a hypothetical new end member of the tourmaline group, with an ideal chemical formula requiring about $20 \mathrm{wt} \% \mathrm{MnO}$. However, "tsilaisite" is not yet officially recognized by mineralogists because no natural material has been found that is at least half composed of the "tsilaisite" component, which would require more than 10.7 wt.\% $\mathrm{MnO}$. Pure "tsilaisite" has reportedly been produced in the laboratory, and provides measurable physical and optical properties. The stone we examined, with its high manganese content, exhibits properties even more like those of the man-made "tsilaisite" than does the material studied by Schmetzer and Bank, and is closer chemically to hypothetical "tsilaisite" than any other tourmaline reported thus far. If gem "tsilaisite" were at some point discovered, it would be distinguishable from gem elbaites by its higher refractive indices, birefringence, and specific gravity. A more detailed discussion of this stone is scheduled to appear in an upcoming issue of the American Mineralogist.

$R K$

\section{FIGURE CREDITS}

The photos used in figures 1,2,14, and 15 were taken by Clayton Welch. Dave Hargett supplied figure 4. John I. Koivula took the photomicrographs used in figures 5, 6, and 18. Tino Hammid is responsible for figure 3. Shane McClure furnished figures $7,10,13,16$, and 20. Chuck Fryer's photos were used in figures 8 and 9 . Bob Crowningshield provided the $X$-radiographs for figures 11 and 12. The absorption spectrum in figure 17 is the work of Bob Kane. Mike Havslad supplied figure 19. 